\title{
Attitudes of $K$-theory
}

Topological, Algebraic, Combinatorial



Inna Zakharevich 


\section{Introduction: Divide and Conquer}

In many areas of mathematics we use similar methods for analyzing problems. One of the most common is known as "divide and conquer": split up a problem into smaller problems, solve each of the smaller problems, then glue the solutions back together into a solution to the whole thing. Often, at the end, there is a frustrating question left over:

Is the constructed solution the only one possible?

In other words, do the parts of the solution uniquely determine the solution to the whole?

One approach to solving this question is that of considering only locally defined objects. For example, if two functions agree at all points then they are equal; thus the answer to the question is always "yes." Sometimes, however, this is not possible, and it is necessary to analyze all of the different ways that pieces can be put together. For example, even if it is known that an abelian group has a filtration with associated graded $\mathbf{Z} / 2 \oplus \mathbf{Z} / 2$, there are multiple possibilities for what the group could be: $\mathbf{Z} / 2 \oplus \mathbf{Z} / 2$ or $\mathbf{Z} / 4$. This is where $K$-theory comes in.

$K$-theory is the study of invariants of assembly problems. Topological $K$-theory studies how vector bundles are assembled; algebraic $K$-theory studies how modules over a ring are assembled; other kinds of $K$-theory study how other kinds of objects can be assembled. The general approach is to define a group $K_{0}$ with a generator for each object of study and a relation for each possible "assembly." Because different areas of mathematics have different tools and approaches, the question of classifying different forms of $K$-theory varies drastically in difficulty from field to field.

In this article we give a brief overview of three different kinds of $K$-theories: topological, algebraic, and combinatorial. Between the sections we give some context and background to explain how the modern perspective on $K$ theory developed, and some of the motivation for the theory behind it. Although this article is not (and cannot) be a comprehensive history of or motivation for the topic, we hope that it will be an interesting perspective on the development of this field.

\section{Topological $K$-Theory}

The first example of $K$-theory that most people see is topological $K$-theory. The idea behind topological $K$-theory is

Inna Zakharevich is an assistant professor of mathematics at Cornell University. Her email address is zakh@math . corne11. edu.

The author was supported in part by NSF grant DMS-1654522.

Communicated by Notices Associate Editor Daniel Krashen.

For permission to reprint this article, please contact:

reprint-permission@ams.org.

DOI: https://doi.org/10.1090/noti1908 to study a (compact Hausdorff) space by examining the ways that vector bundles on that space behave. A vector bundle on a space $X$ is a continuous family of vector spaces indexed by $X$. Somewhat more precisely, it is a space $E$ together with a map $p: E \longrightarrow X$, such that for any $x \in$ $X, p^{-1}(x)$ is a vector space (and all of these fit together nicely). We call $p^{-1}(x)$ the fiber over $x$, and denote it $E_{x}$. These $E_{X}$ are exactly the vector spaces in our continuous family.

We can also think of vector bundles more locally. An example of a vector bundle on $X$ is the "trivial bundle" $X \times \mathbf{R}^{n}$. A general vector bundle looks like a trivial bundle in a neighborhood of any point. Thus we can assemble a bundle by taking several of these and gluing them together. More precisely, we can cover $X$ by a family of open subsets $\left\{U_{\alpha}\right\}_{\alpha \in A}$, and think of the vector bundle as looking like $U_{\alpha} \times \mathbf{R}^{n}$ over each $U_{\alpha}$. We then "glue together" these bundles into a bundle on $X$ similarly to how manifolds are glued together: by giving gluing data that tell us how to identify the part of $U_{\alpha} \times \mathbf{R}^{n}$ that sits over $U_{\alpha} \cap U_{\beta}$ to the part of $U_{\beta} \times \mathbf{R}^{n}$ that sits over $U_{\alpha} \cap U_{\beta}$. One requires that this gluing data should restrict to a linear isomorphism from the copy of $\mathbf{R}^{n}$ over $x$ in $U_{\alpha}$ to the copy of $\mathbf{R}^{n}$ over $x$ in $U_{\beta}$; this gives an element of $\mathrm{GL}_{n}(\mathbf{R})$ for every $x \in U_{\alpha} \cap U_{\beta}$. This gluing data should be continuous; this means that we want continuous maps $g_{\alpha \beta}$ : $U_{\alpha} \cap U_{\beta} \longrightarrow \mathrm{GL}_{n}(\mathbf{R})$ for all $\alpha, \beta$. Assuming that these satisfy some standard relations (for example, that $g_{\alpha \beta}(x)=$ $g_{\beta \alpha}(x)^{-1}$ for all $\left.x \in U_{\alpha} \cap U_{\beta}\right)$ this gives enough data to construct the vector bundle $E$. (For a more precise treatment, see for example [11, Chapter 1].)

Two vector bundles $p: E \longrightarrow X$ and $p^{\prime}: E^{\prime} \longrightarrow X$ are considered isomorphic if there is a homeomorphism $f: E \longrightarrow E^{\prime}$ such that for all $x \in X$, the restriction of $f$ to $E_{X}$ gives a linear isomorphism to $E_{X}^{\prime}$. In other words, $f$ must satisfy $p^{\prime} \circ f=p$.

Let us consider an example. Suppose that $X=S^{1}$ and $n=1$. To each point in $S^{1}$ we attach a line, in such a way that these lines form a continuous family; thus we can think of this as attaching an infinitely wide strip of paper to a circle. Starting at a point in the circle, let us walk around clockwise and try to make sure that the paper is standing up "vertically" (compared to the plane of the circle). When we get back to the beginning one of two things can happen: either the entire strip is standing up vertically (in which case we have the bundle $S^{1} \times \mathbf{R}^{1}$ ) or we failed: there is a twist in the paper, so that we have a Möbius strip. These are the two possibilities for one-dimensional vector bundles on a circle.

A vector bundle is fundamentally a geometric object, but it turns out to be classified by homotopical information. For a concrete example, let us consider $X=S^{n}$ and cover it by the family $\left\{S^{n} \backslash\{\right.$ north pole $\}, S^{n} \backslash\{$ south pole $\left.\}\right\}$. 
An $n$-dimensional vector bundle on $X$ is then uniquely determined by a single function $S^{n} \backslash$ \{poles $\longrightarrow \mathrm{GL}_{n}(\mathbf{R})$. It turns out that the isomorphism class of the vector bundle depends only on the homotopy class of this map, not on the actual map; in particular, we can consider it to be a map from $S^{n-1} \longrightarrow \mathrm{GL}_{n}(\mathbf{R})$ (see [11, Section 1.2]). For example, applying this to the circle case above we see that we have four homotopy classes of maps $S^{0}=\{ \pm 1\}$ $\longrightarrow \mathrm{GL}_{1}(\mathbf{R})$, depending only on the sign of the image of each of the two points. By changing the orientation on one of the two patches, we can assume that the point 1 is mapped to the identity transformation, which gives us two possibilities: if the image of -1 is negative in $\mathrm{GL}_{1}(\mathbf{R})$ then we get a Möbius strip, and otherwise we get the trivial bundle. This confirms our above intuitive description of the possible bundles on $S^{1}$.

With this perspective we see that the geometric data of the vector bundle is controlled by the homotopical data of the gluing maps. In fact, this kind of construction works more generally. Let $\operatorname{Vect}^{n}(X)$ be the set of isomorphism classes of vector bundles on $X$.

Theorem 1 ([11, Theorem 1.16]). There exists a space called $B O(n)$ such that for finite $C W$ complexes $^{1} X$,

$$
\operatorname{Vect}^{n}(X)=[X, B O(n)]
$$

where the right-hand side denotes the homotopy classes of maps $X \longrightarrow B O(n)$.

One way of understanding this theorem is to say, as we did above, that the geometric data of vector bundles is controlled by homotopical data; another way is to say that the geometric data of $\operatorname{Vect}^{n}(X)$ contains homotopical information about $X$.

We can develop this idea into a very powerful theory called topological K-theory.

Let us consider the set of isomorphism classes of vector bundles on $X$, which we write $\operatorname{Vect}(X)$. This set has two operations on it: $\oplus$ and $\otimes$. For any two vector bundles $E, E^{\prime}$ over $X$ we can construct a vector bundle $E \oplus E^{\prime}$ where we set $\left(E \oplus E^{\prime}\right)_{X}=E_{X} \oplus E_{X}^{\prime}$, or a vector bundle $E \otimes E^{\prime}$ by $\left(E \otimes E^{\prime}\right)_{X}=E_{X} \otimes E_{X}^{\prime}$. These two operations are unital: the 0 -dimensional bundle $X \times \mathbf{R}^{0}$ is the unit for $\oplus$, and the 1 -dimensional bundle $X \times \mathbf{R}^{1}$ is the unit for $\otimes$. Thus the only thing stopping us from having a ring of vector bundles on $X$ is that we have no additive inverses.

The solution to this is to add them formally, the same way as we build the rational numbers from the integers. Consider a pair $\left(E, E^{\prime}\right) \in \operatorname{Vect}(X)^{2}$, which we think of as a "formal difference" $E-E^{\prime}$. We define an equivalence relation $\left(E, E^{\prime}\right) \sim\left(F, F^{\prime}\right)$ if $E \oplus F^{\prime} \cong E^{\prime} \oplus F$, exactly analogously to how two fractions $\frac{a}{b}$ and $\frac{c}{d}$ are equal if $a d=b c$.

\footnotetext{
${ }^{1}$ Actually, all that is required is that $X$ is paracompact.
}

We can then define addition by

$$
\left(E, E^{\prime}\right)+\left(F, F^{\prime}\right)=\left(E \oplus F, E^{\prime} \oplus F^{\prime}\right)
$$

and multiplication by

$\left(E, E^{\prime}\right)\left(F, F^{\prime}\right)=\left((E \otimes F) \oplus\left(E^{\prime} \otimes F^{\prime}\right),\left(E \otimes F^{\prime}\right) \oplus\left(E^{\prime} \otimes F\right)\right.$.

These operations define a ring structure on

$$
K O^{0}(X) \stackrel{\text { def }}{=} \operatorname{Vect}(X)^{2} / \sim .
$$

(The group $K^{0}(X)$ is defined analogously using complex vector bundles.)

It turns out [11, Chapter 2] that $K O^{0}(X)$ is a homotopy invariant. Moreover, if we define

$$
K O^{-n}(X) \stackrel{\text { def }}{=} K^{0}\left(\Sigma^{n}\left(X_{+}\right)\right) \quad n \geq 0
$$

(where $\Sigma^{n}\left(X_{+}\right)$is the reduced suspension of $X$ with a disjoint basepoint added [12, Example 0.10]) it turns out that these groups have an 8 -fold periodicity. (This is called the Bott periodicity theorem [5, 1.15].) This allows us to extend this definition to all integers $n$. With these definitions $K O^{*}(X)$ has been used to great advantage to solve various geometric problems. The two most famous are the following:

Hopf invariant 1: There are some standard examples of spheres that have unital multiplications, starting with $S^{0}$ and $S^{1}$, which both have abelian group structures. By noting that $S^{0}$ is the elements of norm 1 in $\mathbf{R}$ and $S^{1}$ is the elements of norm 1 in $\mathbf{C}$ we can construct similar multiplications on $S^{3}$ and $S^{7}$, viewing them as the units in the quaternions and octonions. It turns out that these are the only examples of spheres with unital multiplication; this was originally proved by Adams in [1] using the Steenrod algebra, but a much simpler and cleaner proof was discovered by Adams and Atiyah [3] using topological $K$-theory.

This problem is closely related to other classical questions, including the existence of normed division algebra structures on $\mathbf{R}^{n}$, the parallelizability of $S^{n-1}$, and the existence of spaces with (graded commutative) polynomial cohomology.

Counting vector fields on spheres: The hairy ball theorem states that there is no nonvanishing continuous vector field on $S^{2}$. However, for odd-dimensional spheres such fields exist, and so the more complicated question becomes: what is the maximum number of everywhere-linearly-independent vector fields that can be put on a sphere? Adams [2] showed, using topological $K$-theory, that there are $\rho(n)-1$ such fields on $S^{n-1}$, where $\rho(n)$ is the Radon-Hurwitz number. (Prior to Adams it was known that there are at least that many fields; using the structure of topological $K$ theory Adams was able to show that this is a sharp bound.) 
It turns out that topological $K$-theory is a generalized cohomology theory: a way of assigning groups to spaces that behaves just like singular cohomology, but which does not satisfy the dimension axiom. ${ }^{2}$ Thus all of the standard computational tools that work for ordinary cohomology theory work for topological $K$-theory. For example, for a sub-CW-complex $Y$ of a finite $\mathrm{CW}$-complex $X$ there exists a long exact sequence

$$
\begin{aligned}
\cdots & \longrightarrow K O^{n}(X / Y) \longrightarrow K O^{n}(X) \longrightarrow K O^{n}(Y) \\
& \longrightarrow K O^{n+1}(X / Y) \longrightarrow \cdots .
\end{aligned}
$$

These new invariants, together with their geometric underpinnings, are what give topological $K$-theory its power.

\section{Segue: Representable Cohomology Theories}

The group $\mathrm{KO}^{0}(X)$ was defined algebraically in the previous section, but it is in fact possible to define it completely homotopically. If we write $[X, Y]$ for the set of (basepointed) homotopy classes of maps from $X$ to $Y$, it turns out that

$$
K O^{0}(X) \cong\left[X_{+}, \mathbf{Z} \times B O\right],
$$

where $X_{+}$is $X$ with a disjoint basepoint added, and $B O$ is the delooping ${ }^{3}$ of the infinite orthogonal group (in which elements are orthogonal matrices that differ from the identity in only a finite number of spaces) [11, Section 1.2]. The space $B O$ is closely related to the spaces $B O(n)$ mentioned above; the difference is that previously we were considering vector bundles of a fixed dimension, while here we consider all vector bundles at once. Using the above expression we can then see that

$$
K O^{-n}(X) \cong\left[\Sigma^{n}\left(X_{+}\right), \mathbf{Z} \times B O\right] \cong\left[X_{+}, \Omega^{n}(\mathbf{Z} \times B O)\right],
$$

where $\Omega Y$ is the loop space of $Y$, whose points are (basepointed) maps $S^{1} \longrightarrow Y$. Bott periodicity simply states that

$$
\Omega^{8}(\mathbf{Z} \times B O) \simeq \mathbf{Z} \times B O .
$$

Using these results we can construct a sequence of spaces

$$
\ldots, K_{-2}, K_{-1}, K_{0}, K_{1}, K_{2}, \ldots
$$

such that $K O^{n}(X) \cong\left[X_{+}, K_{n}\right]$.

A similar statement can be made for ordinary cohomology theory. Letting $E_{n}=K(\mathbf{Z}, n)$ be the $n$-th EilenbergMacLane space of $\mathbf{Z}\left[12\right.$, Section 4.2], and setting $E_{n}=*$ for $n<0$ we have

$$
H^{n}(X ; \mathbf{Z}) \cong\left[X_{+}, E_{n}\right] .
$$

\footnotetext{
${ }^{2}$ The dimension axiom states that all cohomology groups of a point other than the 0 -th one must be 0 . This is what allows us to read off the "dimension" of a space from its cohomology. Topological K-theory doesn't work this way: the topological K-theory of a point has infinitely many nonzero groups.

${ }^{3}$ The delooping of a space $Y$ is a space $Z$ such that $Y \simeq \Omega Z$. The delooping of a discrete group is just the classifying space of the group; however, since $O$ has a nondiscrete topology a bit more care is necessary.
}

In fact, the Brown Representability Theorem [6] states that such a construction is possible for any generalized cohomology theory. It thus makes sense to think of a cohomology theory not as a sequence of functors, but instead as a sequence of spaces. The only extra data necessary to produce a cohomology theory from a sequence of spaces is structure maps, which are weak equivalences $X_{n} \stackrel{\sim}{\longrightarrow} \Omega X_{n+1}$. Given this extra data, it makes sense to drop all spaces with negative indices and assume that

$$
X_{-n} \simeq \Omega^{n} X_{0} \quad \text { for } n>0 .
$$

A space $X_{0}$ that can appear at the beginning of such a sequence is called an infinite-loop space, since it is weakly equivalent to an $n$-th loop space for all $n$. In this case, the groups $\pi_{n} X_{0}$ are exactly the cohomology groups of the sphere in the cohomology theory represented by this sequence.

It is common, therefore, to shift perspective from constructing a cohomology theory to constructing a space (which turns out to be an infinite-loop space) whose homotopy groups are exactly the cohomology groups of the sphere (for the cohomology theory in question). Such a space contains all of the information of the cohomology theory, but has much more structure to work with.

\section{Algebraic $K$-Theory}

The next step is to construct a version of $K$-theory for rings, rather than spaces. It is important to note that topological and algebraic $K$-theory are completely distinct, both in approach and results: topological $K$-theory starts with a space and constructs a ring, while algebraic $K$-theory starts with a ring and constructs a space. The connection between them is the spirit of the Serre-Swan theorem:

Theorem 2 (Serre-Swan). The category of real vector bundles over a compact $X$ is equivalent to the category of finitely generated projective modules over the ring $C(X)$ of continuous real-valued functions on $X$.

This theorem is motivation for starting with the following analogy: vector bundles over a space are analogous to finitely generated projective modules over a ring.

We can rewrite the definition of $K O^{0}(X)$ to be the free abelian group generated by vector bundles over $X$, modulo the relation that $\left[E \oplus E^{\prime}\right]=[E]+\left[E^{\prime}\right]$. Analogously, we define the group $K_{0}(R)$ to be the group generated by isomorphism classes of finitely generated projective $R$-modules, modulo the relation that $[A \oplus B]=$ $[A]+[B]$. At this point, when working with topological $K$-theory, we were done: we could use suspension to define the negative $K$-groups, and Bott periodicity handled the rest. Unfortunately, an analogous definition generally produces no new useful groups, and no Bott periodicity; thus a different approach is needed in this situation. 
The Serre-Swan theorem also hints that we must change our grading, from cohomological to homological. Given a map $Y \longrightarrow X$ of spaces, the induced map on rings of functions goes in the opposite direction: $C(X) \longrightarrow C(Y)$. The same will hold for our $K$-theories: previously, if we had a map $Y \longrightarrow X$ it induced a map $K^{0}(X) \longrightarrow K^{0}(Y)$ by pulling back vector bundles. Now, on the other hand, we have the opposite map: given a homomorphism $S \longrightarrow R$ of rings we get a map $K_{0}(S) \longrightarrow K_{0}(R)$ by tensoring an $S$-module up to an $R$-module. In addition, this switch reverses the signs of the $K$-groups, with $K_{1}(R)$ playing an analogous role to $K O^{-1}(X)$.

Topological $K$-theory is a generalized cohomology theory, whose defining property is the existence of a long exact sequence. We are going to keep this, as well as the definition of $K_{0}$. The imagined leap of faith is the observation that long exact sequences appear naturally for the homotopy groups of topological spaces. This is what we are going to construct: given $R$, we want to have a topological space $\mathbf{K}(R)$ and define $K_{i}(R) \stackrel{\text { def }}{=} \pi_{i} \mathbf{K}(R)$. This space should have the property that $\pi_{0} \mathrm{~K}(R) \cong K_{0}(R)$, and such that its higher homotopy groups are meaningful algebraic invariants; in particular, at $K_{1}(R)$ and $K_{2}(R)$ they should agree with classically-defined invariants (for more on this, see [22, Chapters II, III]).

One can see that $K_{0}(R)$ encodes $R$-modules up to certain transformations: isomorphism and stability. Thus $\pi_{1} \mathbf{K}(R)$ should encode loops of such transformations. Let us consider isomorphisms first. If we think of a path between modules as an isomorphism between them, then a loop of such transformations is simply an automorphism of a module. To make this into a group we need to think of the automorphisms of $R$-modules as all living in the same place; fortunately, all finitely generated projective modules can be thought of as summands of the same module, $R^{\infty}$. An automorphism of a module $M$ sitting inside $M \oplus N$ can be thought of as an automorphism of $M \oplus N$ preserving the summands and trivial on $N$. Thus the group of automorphisms we are concerned with is $G L(R)$, the group of infinite invertible matrices with entries in $R$ which are not equal to the identity only within a finite region. This also partially captures the necessary stability.

Since composition of paths of isomorphisms matches multiplication in $G L(R)$, we have a homomorphism $p$ : $G L(R) \longrightarrow K_{1}(R)$. It turns out that stability also implies that $p$ factors through $G L(R)^{\mathrm{ab}}$. Indeed, an element in $G L(R)$ can be represented by a finite matrix $A$ by picking a preimage under an inclusion $G L_{n}(R) \longrightarrow G L(R)$. Because of stability, for a given finite matrix $A$, we must have

$$
\begin{aligned}
& p(A \oplus I)=p(A)=p(I \oplus A) . \text { Thus we must have } \\
& \begin{aligned}
p(A) p(B) & =p(A \oplus I) p(I \oplus B)=p((A \oplus I)(I \oplus B)) \\
& =p(\operatorname{diag}(A, B))
\end{aligned}
\end{aligned}
$$

and analogously $p(A) p(B)=p(\operatorname{diag}(B, A)$. But by this same argument, $p(B) p(A)=p(\operatorname{diag}(A, B))$, so $K_{1}(R)$ must be abelian. It turns out that defining

$$
K_{1}(R) \stackrel{\text { def }}{=} G L(R)^{\mathrm{ab}}
$$

produces a stable invariant. When $R$ is a field this is just the group of invertible elements in $R$, and the map $G L(R)$ $\longrightarrow K_{1}(R)$ takes the determinant of a matrix.

Example. To see that this is a reasonable definition, at least for fields, let us consider the example when $R$ is a Dedekind domain with fraction field $F$. In this case [22, Corollary II.2.6.3] we have $K_{0}(R) \cong \mathrm{Z} \oplus \mathrm{Cl}(R)$, where $\mathrm{Cl}(R)$ is the class group of $R$. Since $K_{0}(F) \cong \mathbf{Z}$, the definition of $\mathrm{Cl}(R)$ leads to an exact sequence

$$
\begin{aligned}
R^{\times} \longrightarrow F^{\times} \longrightarrow \bigoplus_{p \text { prime }} \mathrm{Z} \longrightarrow K_{0}(R) \longrightarrow K_{0}(F) \\
\longrightarrow 0 .
\end{aligned}
$$

Note that each term in the infinite sum is actually $K_{0}(R / \mathfrak{p})$. The determinant gives a surjective map $G L(R) \longrightarrow R^{\times}$, so in fact there is a surjective map $K_{1}(R) \longrightarrow R^{\times}$. We can therefore rewrite the above sequence as

$$
\begin{aligned}
K_{1}(R) & \longrightarrow K_{1}(F) \longrightarrow \bigoplus_{\mathfrak{p} \text { prime }} K_{0}(R / \mathfrak{p}) \longrightarrow K_{0}(R) \\
& \longrightarrow K_{0}(F) \longrightarrow 0 .
\end{aligned}
$$

This may be considered another confirmation of the correctness of our definition of $K_{1}(R){ }^{4}$

We now turn to a construction of $\mathbf{K}(R)$. A naive way to begin is to try and construct a CW complex whose points are represented by finitely generated projective $R$-modules, with higher cells matching relations on $K_{0}$. However, this is difficult because of the nature of the relation on $K_{0}$ : it is a three-term relation. Adding higher cells between 0 cells can impose two-term relations, setting things equal to one another, but it is much more difficult to impose a three-term relation. One way to attempt this is to add points labelled by formal sums of projective $R$-modules. However, the more fruitful approach is to shift perspective: a three-term relation can be represented by a triangle; thus constructing a space where arcs represent projective $R$ modules and triangles represent relations is a more natural approach. As this produces a space whose $\pi_{1}$ is $K_{0}(R)$, we end our construction by taking a loop space. One extra advantage of this approach is that we no longer need to worry

\footnotetext{
${ }^{4}$ In fact, historically speaking, we are doing this backwards. The definitions of $K_{0}, K_{1}$, and $K_{2}$ came first; they were then observed to fit into such an exact sequence, and the idea of thinking of them as the homotopy groups of a space came later.
} 
about constructing "negatives" of $R$-modules: since each module is represented by a loop, its negative is simply the same loop, traversed backwards.

We begin the construction with a single point. We then add an arc for every finitely generated projective $R$-module. We then add a cell for each relation. It is important to note that we need a relation for every way of assembling $A \oplus B$ from $A$ and $B$; the best way to keep track of these is to label relations by exact sequences

$$
0 \longrightarrow A \longrightarrow A \oplus B \longrightarrow B \longrightarrow 0 \text {. }
$$

We now have the correct $\pi_{1}$, but our higher homotopy groups are far too large: many of these exact sequences have relations between them. We just need to figure out what such relations may look like.

Already the case when $R$ is a field is highly nontrivial. For example, $K_{*}(\mathrm{C})$ is known completely only for finite coefficients [22, Section VI.1]. Suppose that we are given a vector space $V$ of dimension $a+b+c$. We wish to decompose it into spaces of dimensions $a, b$, and $c$. We can do this by selecting a subspace of dimension $a$, then quotienting to get a space of dimension $b+c$, then picking a subspace of that of dimension $b$ and quotienting for the space of dimension $c$. Alternately, we could pick a subspace of dimension $a+b$, quotient to get a subspace of dimension $c$, and then decompose the subspace of dimension $a+b$. These ways of getting a subspace decomposition should, in some real sense, be equivalent.

There are several ways of correctly encoding such equivalences. The most general is to shift our perspective on exact sequences: instead of thinking of an exact sequence as a way of decomposing $A \oplus B$ into $A$ and $B$, we instead think of it as a filtered ${ }^{5} R$-module $A \oplus B$ with only two filtered layers. The three-term relation then says that the whole module is equal to the first filtration plus the quotient. This gives us a method for inserting new relations: extending the length of the filtration. Given a filtered $R$-module $A_{1} \subseteq A_{2} \subseteq A_{3}$, the three-term relation can break down $A_{3}$ in two different ways. First, we could break down $A_{3}$ as $A_{2}$ and $A_{3} / A_{2}$ and break down $A_{2}$ as $A_{2} / A_{1}$ and $A_{1}$. Alternately, we could break down $A_{3}$ into $A_{3} / A_{1}$ and $A_{1}$, and then further break $A_{3} / A_{1}$ as $\left(A_{3} / A_{1}\right) /\left(A_{2} / A_{1}\right) \cong$ $A_{3} / A_{2}$ and $A_{2} / A_{1}$. These two ways of decomposing $A_{3}$ should be equivalent, as both decompose $A_{3}$ into $A_{1}$, $A_{2} / A_{1}$, and $A_{3} / A_{2}$. Such a filtered module should therefore impose a four-term relation

$$
\begin{aligned}
& {\left[A_{1} \subseteq A_{2}\right]+\left[A_{2} \subseteq A_{3}\right] } \\
&=\left[A_{2} / A_{1} \subseteq A_{3} / A_{1}\right]+\left[A_{1} \subseteq A_{3}\right]
\end{aligned}
$$

\footnotetext{
${ }^{5}$ We need a little bit more structure to make this work correctly; instead of taking just a filtration $M_{0} \subseteq \cdots \subseteq M_{n}$ we must take a sequence $M_{0} \hookrightarrow \cdots \hookrightarrow M_{n}$ of inclusions. In particular, isomorphisms $M_{0} \stackrel{\cong}{\longleftrightarrow} M_{1}$ are allowed, and different choices of isomorphism are assigned to different cells.
}

We can add a 3-cell labelled by each triple of projective $R$ modules via a gluing map corresponding to this equation. There are now relations that are imposed by four-term filtered $R$-modules, and five-term filtered $R$-modules, and so on; we thus produce an infinite-dimensional CW complex that is called $\left|s_{\bullet} \mathbf{P}_{R}\right|$; as we shall see in the next section, this is a special case of a construction called the Waldhausen $S_{\bullet}$ construction [20, Section 1.4]. ${ }^{6}$ To get the $K$-theory with the correct $\pi_{0}$ we take its loop space, shifting the homotopy groups back down a dimension:

$$
\mathbf{K}(R) \stackrel{\text { def }}{=} \Omega\left|S_{\bullet} \mathbf{P}_{R}\right| .
$$

To get the higher $K$-groups we define

$$
K_{n}(R) \stackrel{\text { def }}{=} \pi_{n} \mathbf{K}(R) .
$$

By construction this gives the correct $K_{0}$. Proving that this gives $\mathrm{GL}(R)^{\mathrm{ab}}$ on $\pi_{1}$ is more difficult; for details on this see [22, Exact Categories IV.8.6, Corollary IV.7.2].

Algebraic $K$-theory is very mysterious: it is famously difficult to compute and is related to many important invariants. For example, the Quillen-Lichtenbaum conjecture (proved by Vladimir Voevodsky) states that there should be a spectral sequence beginning at étale cohomology of Spec $R$ and converging to the $K$-theory of $R$. Assuming the Kummer-Vandiver conjecture (which is a statement about the class numbers of cyclotomic fields) [21, Theorem 1] the $K$-theory of the integers was computed to be $K_{0}(\mathbf{Z}) \cong \mathbf{Z}, K_{1}(\mathbf{Z}) \cong \mathbf{Z} / 2$, and

$$
K_{n}(\mathbf{Z}) \cong \begin{cases}0 & \text { if } n>0 \text { and } n \equiv 0(\bmod 4) \\ \mathbf{Z} \oplus \mathbf{Z} / 2 & \text { if } n \equiv 1(\bmod 8) \text { and } n>1 \\ \mathbf{Z} / c_{k} \oplus \mathbf{Z} / 2 & \text { if } n \equiv 2(\bmod 8) \\ \mathbf{Z} / 8 d_{k} & \text { if } n \equiv 3(\bmod 8) \\ \mathbf{Z} & \text { if } n \equiv 5(\bmod 8) \\ \mathbf{Z} / c_{k} & \text { if } n \equiv 6(\bmod 8) \\ \mathbf{Z} / 4 d_{k} & \text { if } n \equiv 7(\bmod 8),\end{cases}
$$

where $c_{k} / d_{k}$ is the Bernoulli number $B_{2 k} / k$ in lowest terms and $n=4 k-1$ or $4 k-2$. The most famous computation of algebraic $K$-theory is still Quillen's original computation of the $K$-theory of finite fields [14], which has since strongly resisted generalizations. More modern computational techniques use close approximations to $K$-theory, such as $T H H$ and $T C$; for more on this see for example [9].

\footnotetext{
${ }^{6}$ This construction is one of two constructions in Waldhausen's paper, called the $S_{\bullet}$ construction and the $S_{\bullet}$-construction. The difference between them is that the $S_{\bullet}$ construction can also incorporate a notion of "weak equivalence."
} 


\section{Intermezzo: Categories Representing Spaces}

The close connection between algebraic topology and category theory is often presented historically: many of the fundamentals of category theory were developed by homotopy theorists in connection with homology and cohomology. Here we pursue a different approach illustrating the importance of categories in homotopy theory, with the following slogan:

Categories give algebraic models for homotopy types of spaces, analogously to the way that rings of functions give algebraic models for geometric objects.

This point of view clarifies why category theory is so intimately intertwined with homotopy theory. To substantiate the claims above, let us explore the basic constructions. This is a very informal introduction; for a more complete treatment see, for example, [16].

A category is a collection of objects, together with a collection of morphisms between objects. A morphism has a source and a target; a morphism with source $A$ and target $B$ is written $f: A \longrightarrow B$. Morphisms have an operation of composition: given a morphism $f: A \longrightarrow B$ and a morphism $g: B \longrightarrow C$ there is a morphism $g \circ f: A \longrightarrow C$.

Some examples of categories:

1. The category Set has sets as objects and functions as morphisms between sets (with the usual composition). One can similarly define categories of groups and group homomorphisms, topological spaces and continuous maps, smooth manifolds and smooth maps, etc.

2. Suppose that we are given a partial order $(P, \leq)$. We can define a category with objects the elements of $P$, and with exactly one morphism $a \longrightarrow b$ if $a \leq b$. Composition is well-defined by transitivity of $\leq$.

3. Let $G$ be a group. We define a category with one object $*$, and with the set of morphisms $* \longrightarrow *$ given by the elements of $G$. Composition is the multiplication in $G$.

Given a category $C$ for which objects form a set, we can define the classifying space $|C|$. Take a 0 -cell for every object in the category. For every morphism $A \longrightarrow B$ attach a 1-cell between the 0 -cell for $A$ and the 0 -cell for $B$. For every pair of composable morphisms $f: A \longrightarrow B$ and $g: B \longrightarrow C$ attach a 2 -cell along $f$, then $g$, and then backwards along $g \circ f$. For every triple of composable morphisms $f: A \longrightarrow B, g: B \longrightarrow C, h: C \longrightarrow D$ attach a 3-cell by thinking of it as a tetrahedron and gluing it in along the triangles shown below:

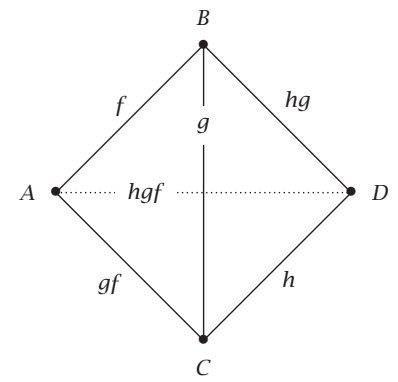

Continue likewise for all $n$-tuples of composable morphisms. ${ }^{7}$ In the case when the category is obtained from a group $G$ this construction produces the usual classifying space $B G$ of the group. In the case when the category comes from a finite partial order $(P, \leq)$, this produces the abstract simplicial complex given by the set of finite totally ordered subsets of $P$. Thus, for example, if $P=\left\{a_{1}, a_{2}, b_{1}, b_{2}\right\}$ with the ordering given by $a_{i} \leq b_{j}$ for $i, j=1,2$, the category obtained from $P$ is drawn on the left, and its classifying space is the circle on the right:
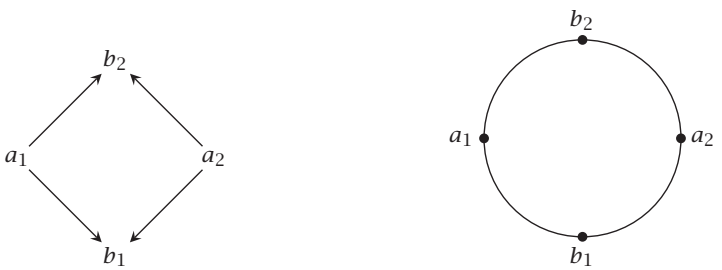

In the slightly more complicated case when $P=\left\{a_{1}, a_{2}, b_{1}, b_{2}, c_{1}, c_{2}\right\}$ with the ordering given by $a_{i} \leq b_{j} \leq c_{k}$ for $i, j, k=1,2$ the category obtained from $P$ is drawn on the left and its classifying space is the sphere on the right:
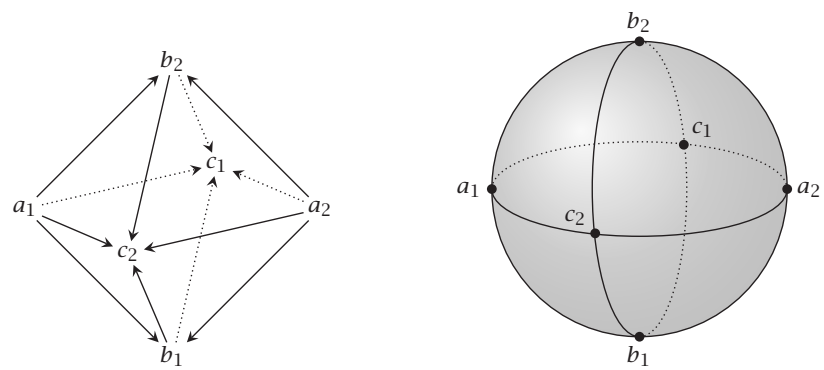

Comparing this to the construction of $\left|s_{\bullet} \mathbf{P}_{R}\right|$ in the previous section, we see that it is almost directly analogous to the construction of the classifying space of a category. However, the $s_{\bullet}$-construction has $n$-simplices labelled by compositions with indexing 1, rather than 0 ; for example, a morphism gives a 1-simplex in the classifying space of a category, but a 2 -simplex in the $S_{\bullet}$-construction. We thus need an extra face to each simplex; this is given by taking $A_{1} \hookrightarrow \cdots \hookrightarrow A_{n}$ to $A_{2} / A_{1} \hookrightarrow \cdots \hookrightarrow A_{n} / A_{1}$.

\footnotetext{
${ }^{7}$ This is somewhat of an oversimplification, as we must deal with identity maps (and morphisms that compose to identity maps) in a special way. This construction is the geometric realization of the nerve of the category; for a more detailed introduction see [16, Chapter 6], as well as [10, Section 1.1].
} 
Before we end this section, let us spend a little time considering how the categorical structure can help model the homotopy class of a space. When working with categories, we do not work with categories up to isomorphism; instead, we work up to equivalence. The idea here is that it should not matter how many different isomorphic models of the same object exist in a category. For example, we can consider the following three categories:

1. The category whose objects are finite subsets of $\mathbf{R}$, with functions as its morphisms.

2. The category whose objects are finite subsets of $\mathbf{Z}$, with functions as its morphisms.

3. The category whose objects are the sets $\{1, \ldots, n\}$ for $n \geq 0$, with functions as its morphisms.

All of these should model "the category of finite sets," and thus should all behave the same way, despite the fact that each one restricts to a subset of the objects of the previous ones. This is the notion of equivalence of categories, and it behaves analogously to the way that homotopy equivalences can "thicken" spaces by, for example, replacing a circle with a solid torus.

We can also use the algebraic structure of a category to understand the homotopy type of its classifying space. For example, a category with an initial object (an object with a unique morphism to every other object in the category, such as the empty set in Set) has a contractible classifying space: the initial object behaves like a "cone point," connecting uniquely to everything else, and allows us to retract every point in the classifying space to it. For those readers familiar with adjoint functors, we state the following theorem, which is a generalization of this observation:

Theorem 3. Given a pair of adjoint functors $F: C \rightleftarrows \mathcal{D}: G$ the induced maps on the classifying spaces give mutually inverse homotopy equivalences. ${ }^{8}$

In fact, it turns out that all homotopical behaviors of topological spaces can be modeled by categories; this observation is due to Thomason [19].

\section{Combinatorial $K$-Theory}

When we constructed algebraic $K$-theory we used almost none of the algebraic information about $R$; all we used is data about how to include and quotient $R$-modules. Thus this same kind of construction can be used with more geometric objects-such as varieties or polytopes-replacing modules to construct a $K$-theory of a more combinatorial nature.

Let us consider the $S_{\bullet}$-construction again and attempt to create a $K$-theory for finite sets. Finite sets are made up of their subsets, in the same way that modules are made up

\footnotetext{
${ }^{8}$ This theorem can be proved as an application of $[15$, Theorem A]. It can also be proved directly from the definition of the nerve; for a quick introduction to simplicial sets and nerves, see for example [17].
}

by submodules (and quotients); thus we begin by considering the disjoint union of finite sets. By analogous logic to the section "Algebraic $K$-theory," this leads to $K_{0}$ (FinSet) $\cong \mathrm{Z}$, with the element $n$ representing a set of size $n$, and $K_{1}$ (FinSet) $\cong \mathrm{Z} / 2$ representing the abelianization of the group of permutations: the sign. Our hope is that the $s_{\bullet}$-construction should give a construction of a $K$-theory space that agrees with these computations.

We consider the simplices in the $s_{\bullet}$-construction. We take an $n$-cell for each filtered finite set $A_{1} \subseteq A_{2} \subseteq \cdots \subseteq$ $A_{n}$ and, thinking of it as an $n$-simplex, want to attach it to the cells labeled $A_{1} \subseteq \cdots \subseteq \hat{A}_{i} \subseteq \cdots \subseteq A_{n}$, as well as $\left(A_{2} / A_{1}\right) \subseteq \cdots \subseteq\left(A_{n} / A_{1}\right)$-but what should we take instead of $/$ ? The analog of taking quotients of modules is takings quotients of sets. In order for our $K_{0}$ to work correctly, we need the 2-cell labeled by $A \subseteq A$ to be attached to $A, A$, and $\varnothing$, to represent the relation $[A]=[A]+[\varnothing]$. However, if we take the quotient of finite sets, we get $[A]=[A]+[$ singleton $]$, which would imply the relation $|A|=|A|+1$ inside $\mathbf{Z}$; clearly not what is intended.

To solve this problem we take a very simple approach: we simply take complements instead of quotients. We label the last face by

$$
\left(A_{2} \backslash A_{1}\right) \subseteq \cdots \subseteq\left(A_{n} \backslash A_{1}\right),
$$

which now gives the correct relations. We call this construction the $\tilde{S}_{\bullet}$-construction. For a more detailed exploration of this construction, see [7]. We can then define

$$
\mathbf{K}(\text { FinSet }) \stackrel{\text { def }}{=} \Omega \mid \tilde{S}_{\bullet} \text { FinSet } \mid .
$$

By the Barratt-Priddy-Quillen Theorem, the $K$-theory of finite sets is the space $Q S^{0}$ (the 0 -space of the sphere spectrum), whose homotopy groups are the stable homotopy groups of spheres (the calculation of which is one of the fundamental unsolved problems in algebraic topology today).

This sets up an interesting parallel. The integers are defined as a group completion of finite sets, with multiplication induced by Cartesian product of sets. The ring of integers is the fundamental object in number theory and commutative algebra. Under $K$-theory, the finite sets (with a multiplicative structure induced by Cartesian product) are related to the sphere spectrum, which is a fundamental object in homotopy theory and derived algebraic constructions. This illustrates that $K$-theory is intrinsically intertwined with the foundations of algebraic topology, providing further evidence that our construction was well chosen.

We can apply the $\tilde{S}_{\bullet}$-construction more generally by looking at other types of objects that have complements. For example, we can consider finite $G$-sets (or almost-finite $G$-sets for a profinite group $G$ ), polytopes in a uniform 
geometry, $O$-minimal structures, or varieties. In each of these the construction produces a $K$-theory in which elements of $K_{0}$ classify (stable) scissors congruence invariants. (For a more in-depth discussion of such examples and their $K$-theory, see $[7,24]$.)

Let us investigate the case of varieties more closely. We take the category whose objects are varieties over a field $k$, with closed immersions as morphisms. The $\tilde{s}_{\bullet}$-construction gives a space $\mathbf{K}\left(\operatorname{Var}_{k}\right)$ where $K_{0}\left(\operatorname{Var}_{k}\right)$ is the free abelian group generated by varieties, modulo the relation that

$$
[X]=[Y]+[X \backslash Y]
$$

whenever $Y$ is a closed subvariety of $X$. This is the Grothendieck ring of varieties. To construct $\mathbf{K}\left(\mathbf{V a r}_{k}\right)$ we can again use the Waldhausen $\tilde{s}_{\bullet}$-construction. For the inclusions we use closed embeddings; for the complements we use the complement of the image of the embedding. ${ }^{9}$ Analogously to the case of finite sets defined above, this produces a space and we can define

$$
\mathbf{K}\left(\operatorname{Var}_{k}\right) \stackrel{\text { def }}{=} \Omega\left|\tilde{s}_{\bullet} \operatorname{Var}_{k}\right| \text {. }
$$

The higher $K$-groups encode invariants of piecewise-automorphisms.

In previous examples, the calculation of $K_{0}$ was simple, and it was the higher $K$-groups that gave interesting invariants related to automorphisms. However, for varieties, even the calculation of $K_{0}$ is extremely nontrivial; it turns out the higher homotopy of $\mathbf{K}\left(\operatorname{Var}_{k}\right)$ can create new tools for the analysis of $K_{0}$.

Consider the filtration by dimension on the set of varieties. For any dimension $n$, we can construct a group $K_{0}\left(\operatorname{Var}_{k}^{\leq n}\right)$ generated by varieties of dimension at most $n$ (with the same relation as above). We can then filter $K_{0}\left(\operatorname{Var}_{k}\right)$ by setting the $n$-th filtration to be the image of the natural map $\iota_{n}: K_{0}\left(\operatorname{Var}_{k}^{\leq n}\right) \longrightarrow K_{0}\left(\operatorname{Var}_{k}\right)$. Unfortunately, since we do not know that $\iota_{n}$ is injective, we cannot use information about $K_{0}\left(\operatorname{Var}_{k}^{\leq n}\right)$ to learn about $K_{0}\left(\operatorname{Var}_{k}\right)$.

Here is where having a space, rather than a set, is crucial. A filtration on a space does not need to produce a filtration on the connected components. Consider, for example, the two-step filtration $\mathbf{Z} \subseteq \mathbf{R}$ of the integers sitting inside the reals; the integers have infinitely many connected components, while the reals only have one, but the filtration is topologically well-defined. In fact, the filtration quotient is a bouquet of circles, which in its fundamental group encodes the data that the filtration joins infinitely many connected components into one. We can therefore hope that, if we can compute the associated grade of the dimension

${ }^{9}$ As in the section "Algebraic $K$-theory," we need to remember the particular immersion that was chosen for each cell in the construction. filtration on the space $\mathbf{K}\left(\mathbf{V a r}_{k}\right)$, we can extract information about the filtration on $K_{0}\left(\operatorname{Var}_{k}\right)$.

From our construction we see that the map $\mathbf{K}\left(\operatorname{Var}_{k}^{\leq n-1}\right) \longrightarrow \mathbf{K}\left(\operatorname{Var}_{k}^{\leq n}\right)$ induces an inclusion of spaces, which allows us to compute the cofiber of this map as a spectrum. ${ }^{10}$ (For a much more detailed discussion of this, see $[8,23,24]$.) In fact, we have the following:

Theorem 4 ([23, Theorem A]). The cofiber of the inclusion $\mathbf{K}\left(\operatorname{Var}_{k}^{\leq n}\right) \hookrightarrow \mathbf{K}\left(\operatorname{Var}_{k}^{\leq n-1}\right)$ is equivalent (as a spectrum) to $\bigvee_{[X] \in B_{n}} \sum^{\infty}\left(B \operatorname{BiratAut}(X)_{+}\right)$. Here, $B_{n}$ is the set of birational isomorphism classes of varieties of dimension $n$, $\operatorname{BiratAut}(X)$ is the group of birational automorphisms of $X$, $\Sigma^{\infty}$ takes the suspension spectrum associated to a space, and $\cdot+$ adds a disjoint basepoint.

In particular, this means that, morally speaking, the spectrum $\mathbf{K}\left(\operatorname{Var}_{k}\right)$ is assembled out of classifying spaces of birational automorphism groups. As a consequence of this we can conclude that all elements in the kernel of $\iota_{n}$ must be in the form $[X \backslash U]-[X \backslash V]$ for some birational automorphism $\varphi: X \rightarrow X$ represented by an isomorphism $U \longrightarrow V$. In this way the automorphism invariants living in $K_{1}$ control what can happen in $K_{0}\left(\operatorname{Var}_{k}\right)$; the higher structure of $\mathbf{K}\left(\operatorname{Var}_{k}\right)$ is fundamentally intertwined with its 0-level structure.

\section{Codla: The Birds-Eye Perspective}

The focus of this article has been on problems arising in algebraic or geometric contexts: vector bundles, projective modules, polytopes, etc. In each context, the moral of the story was that for each such problem there is a $K$-theory space, whose homotopy groups classify higher invariants.

In fact, there is an even more general approach to $K$ theory. Any time that a problem can be modeled by a category $C$ with an operation ${ }^{11}$ (such as finite sets with isomorphisms as morphisms, and disjoint union as the operation) we can construct a topological monoid, since the classifying space $|C|$ of the category inherits an operation from $C$. The $K$-theory is, morally speaking, the group completion of this monoid. There is an interesting theorem of McDuff-Segal:

Theorem 5 ([13]). For a topological monoid $M$, the topologically correct group completion of $M$ is $\Omega B M$; here, $B M$ is a generalization of the delooping of $M$.

For a category $C$ with operation, it therefore makes sense to define

$$
\mathbf{K}(C) \stackrel{\text { def }}{=} \Omega B|C| .
$$

\footnotetext{
${ }^{10}$ For those unfamiliar with spectra, it is possible to mentally replace "spectrum" with "space" in the following discussion. This will not produce correct mathematics, but will be close enough to explain the narrative.

${ }^{11}$ More formally, a symmetric monoidal structure.
} 
This formula describes $\mathbf{K}(C)$ as a group completion, which is defined by its universal properties. Likewise, more general $K$-theory can be defined via a universal property (see [4]).

Unfortunately, this perspective comes with a severe drawback: it is almost impossible to work with. Generally, when constructing $K$-theories, we wish to be able to prove things about them: to compute the higher $K$-groups, to relate them to other forms of $K$-theory, to compute the homotopy fibers or cofibers of maps between $K$-theories, and so on. The definition of $K$-theory given by the McDuffSegal theorem was not designed for such functionality, and thus (unsurprisingly) is not well-suited for such analysis. This is because the $B$ functor in the definition is extremely difficult to work with, and is particularly unsuitable for calculations.

The solution to this is often to find another construction-such as the $S_{\bullet}$-construction [20], Quillen's $Q$-construction [15], or the $B$-construction for $\Gamma$-spaces [18]for the $K$-theory space. While the McDuff-Segal theorem gives a clean and simple definition, these constructions come with many tools that one can use to analyze their results. Thus the study of $K$-theory often sits awkwardly between the formal and the practical, trading off simplicity for usefulness and looking for beauty in between.

ACKNOWLEDGMENT. The author would like to thank Ilya Zakharevich for extensive edits and suggestions which massively improved the quality of exposition. The author would also like to thank the two anonymous referees for their time and assistance in tightening up the exposition even further.

\section{References}

[1] Adams J. On the non-existence of elements of hopf invariant one, Annals of Mathematics, no. 1 (72):20-104, 1960. www.jstor.org/stable/1970147

[2] Adams J. Vector fields on spheres, Ann. of Math. (2) (75):603-632, 1962 . https://doi-org. proxy. 1ibrary.corne11.edu/10.2307/1970213. MR0139178

[3] Adams J, Atiyah M. $K$-theory and the Hopf invariant, Quart. J. Math. Oxford Ser. (2) (17):31-38, 1966, DOI 10.1093/qmath/17.1.31. MR0198460

[4] Blumberg A, Gepner D, Tabuada G. A universal characterization of higher algebraic K-theory, Geom. Topol., no. 2 (17):733-838, 2013, DOI 10.2140/gt.2013.17.733. MR3070515

[5] Bott R. The periodicity theorem for the classical groups and some of its applications, Advances in Math. (4):353411 (1970), 1970, DOI 10.1016/0001-8708(70)90030-7. MR0259904

[6] Brown EH. Cohomology theories, Ann. of Math. (2) (75):467-484, 1962, DOI 10.2307/1970209. MR0138104
[7] Campbell J. The K-theory spectrum of varieties. arXiv:1505.03136.

[8] Campbell J, Zakharevich I. CGW-categories. arXiv: 1811.08014.

[9] Dundas BI, Goodwillie TG, McCarthy R. The local structure of algebraic K-theory, Algebra and Applications, vol. 18, Springer-Verlag London, Ltd., London, 2013. MR3013261

[10] Goerss PG, Jardine JF. Simplicial homotopy theory, Progress in Mathematics, vol. 174, Birkhäuser Verlag, Basel, 1999. MR1711612

[11] Hatcher A. Vector bundles and K-theory.https://www. math.corne11. edu/ hatcher/VBKT/VBpage.htm7

[12] Hatcher A. Algebraic topology, Cambridge University Press, Cambridge, 2002. MR1867354

[13] McDuff D, Segal G. Homology fibrations and the "group-completion" theorem, Invent. Math., no. 3 (31):279-284, 1975/76, DOI 10.1007/BF01403148. MR0402733

[14] Quillen D. On the cohomology and $K$-theory of the general linear groups over a finite field, Ann. of Math. (2) (96):552-586, 1972, DOI 10.2307/1970825. MR0315016

[15] Quillen D. Higher algebraic $K$-theory. I, Algebraic $K$ theory, I: Higher $K$-theories (Proc. Conf., Battelle Memorial Inst., Seattle, Wash., 1972); 1973:85-147. Lecture Notes in Math., Vol. 341. MR0338129

[16] Riehl E. Category theory in context, Aurora: Dover Modern Math Originals, Dover Publications, 2017. https:// books.google.com/books?id=6B9MDgAAQBAJ

[17] Riehl E. A leisurely introduction to simplicial sets. www. math.jhu.edu/ erieh1/ssets.pdf.

[18] Segal G. Categories and cohomology theories, Topology (13):293-312, 1974, DOI 10.1016/0040-9383(74)900226. MR0353298

[19] Thomason R. Cat as a closed model category, Cahiers Topologie Géom. Différentielle, no. 3 (21):305-324, 1980. MR591388

[20] Waldhausen F. Algebraic $K$-theory of spaces, Algebraic and geometric topology (New Brunswick, N.J., 1983); 1985:318-419, DOI 10.1007/BFb0074449. MR802796

[21] Weibel C. Algebraic $K$-theory of rings of integers in local and global fields, Handbook of $K$-theory. Vol. 1, 2; 2005:139-190, DOI 10.1007/3-540-27855-9_5. MR2181823

[22] Weibel CA. The K-book: An Introduction to Algebraic $K$-theory, Graduate Studies in Mathematics, vol. 145, American Mathematical Society, Providence, RI, 2013. MR3076731

[23] Zakharevich I. The annihilator of the Lefschetz motive, Duke Math. J., no. 11 (166):1989-2022, 201708. dx. doi . org/10.1215/00127094-0000016X

[24] Zakharevich I. The $K$-theory of assemblers, Adv. Math. (304):1176-1218, 2017, DOI 10.1016/j.aim.2016.08.045. MR3558230 


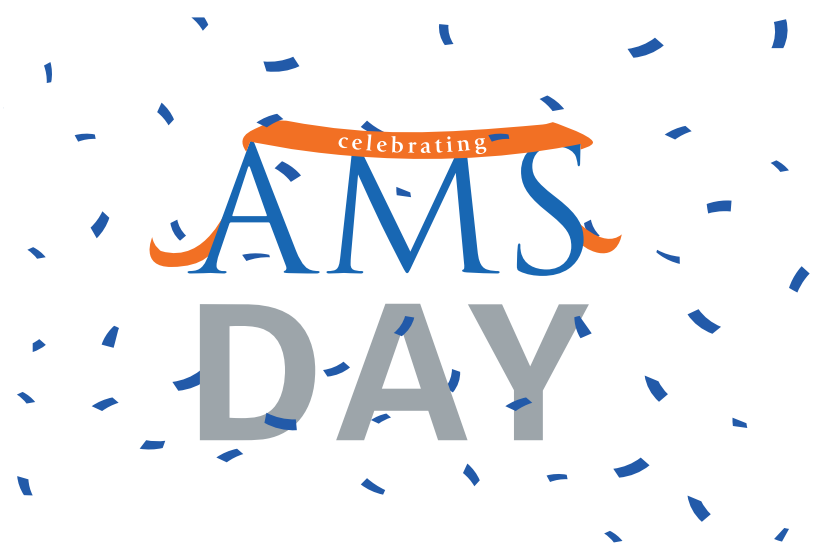

\section{A celebrátion of AMS meimbers!'}

Join us on Wednesday, October 16,

_ 2019 as we honor our AMS members

via "AMS Day", a day of specials on

AMS publications, membership, and

- more! Stay tuned on Facebook, Twitter,

and member emails for details about

this exciting day. Spread the word

- about \#AMSDay today!
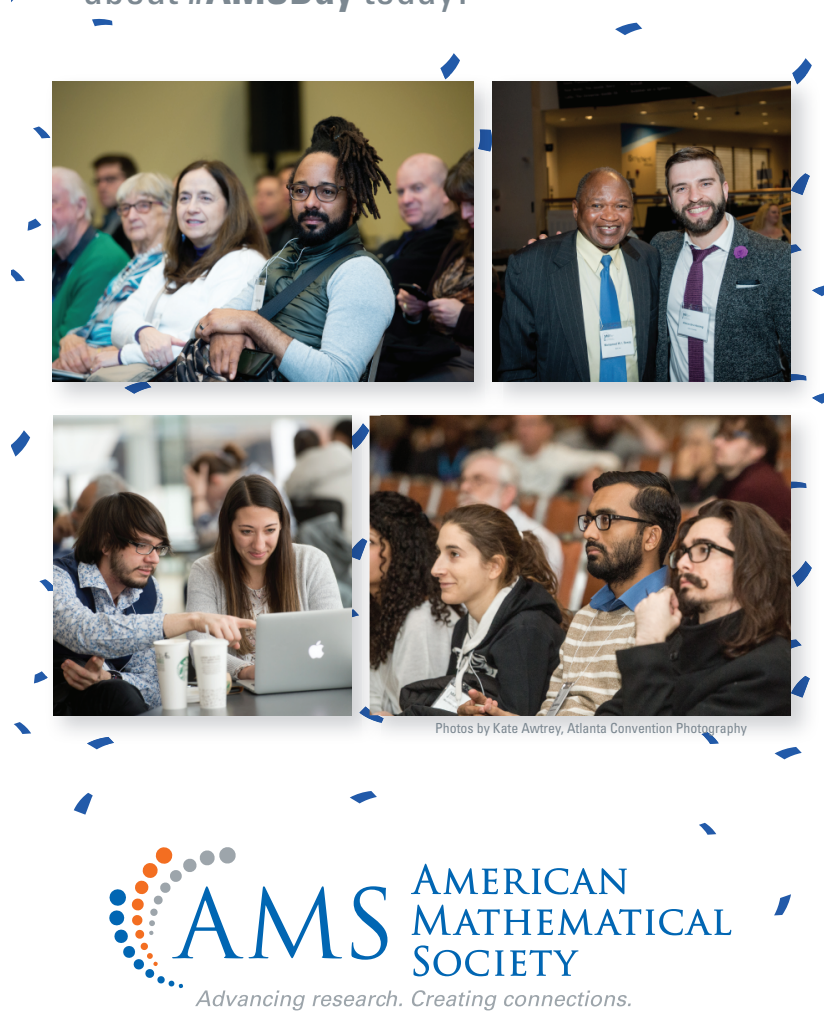

$-$

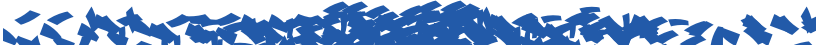

